\title{
LOTTA AL TERRORISMO INTERNAZIONALE TRA STRATEGIA DELLA TENSIONE E VIOLAZIONE DEI DIRITTI UMANI
}

\author{
FIGHTING INTERNATIONAL TERRORISM BETWEEN THE STRATEGY OF THE TENSION \\ AND VIOLATION OF HUMAN RIGHTS
}

Ubaldo Nazzaro

Ph. D. in "Sistema penale integrato e processo" -

Università di Napoli Federico II

E-mail: ubaldonazzaro@hotmail.com

Convidado

\begin{abstract}
L'obiettivo di questo lavoro sulla lotta contro il terrorismo internazionale tra strategia per la tensione e la violazione dei diritti umani è valutare le strategie del controllo globale.

Parole chiave: Terrorismo Internazionale. Flussi Migratori. Violazione dei Diritti Umani.

SOMMARIO: Cenni introduttivi. 1. La mistificazione statunitense della lotta al terrorismo internazionale tra politiche di intervento militare nelle aree di interesse geopolitico e legislazione dell'emergenza. - 2 I provvedimenti comunitari ispiratori della prima legislazione antiterroristica italiana. -3 Misure di contrasto al terrorismo internazionale nella legislazione penale italiana. Riflessioni conclusive. Riferimenti.
\end{abstract}

\section{CENNI INTRODUTTIVI}

All'indomani della Seconda guerra mondiale, le speranze della pace, riposte nell'evoluzione del diritto e della comunità internazionali, furono tradite dalla nascita dell'O.N.U., organismo privo di strumenti idonei alla riduzione degli squilibri economici e finanziari principali cause dei conflitti, che ben presto rivelò la reale natura di organizzazione egemonica nel cui ambito rilievo esclusivo è attribuito ai Paesi vincitori (in primis, gli Stati Uniti) ${ }^{1}$. A soli cinque Stati è attribuito il ruolo di membro permanente del Consiglio di Sicurezza con diritto di veto. La medesima struttura delle Nazioni Unite ne pone in evidenza i connotati di garante della c.d. «pace gerarchica» ${ }^{2}$.

Alla medesima stregua, nella fase successiva al Secondo conflitto mondiale, il percorso comunitario che ha condotto alla genesi dell'U.E. ebbe a individuare nella creazione di un mercato comune la via democratica. Le speranze e le promesse dei padri dell'U.E., che intravedevano in siffatta opzione uno strumento in grado di assicurare la pace e la cooperazione solidale tra i popoli europei, sono state tradite. L'Europa del mercato e dell'Euro finisce, infatti, col prevalere su (rectius, sostituirsi a) quella delle istituzioni democratiche e

\footnotetext{
${ }^{1}$ Cfr. M. DELMAS-MARTY, Studi giuridici comparati e internazionalizzazione del diritto, Torino, 2004, 23; C. FILOSA, L'ONU: mediazione irrigidita. Una pretesa universalità del particolare dominio di classe, in REDLINK (a cura di), L'ONU e «i signori della pace», Milano, 2004, 90 ss.; G. POOLE, Cosa fare dell'ONU?, in REDLINK (a cura di), L'ONU e «i signori della pace», cit., 98 ss.; S. SERINO, Una replica a Gordon Poole, in REDLINK (a cura di), L'ONU e «i signori della pace», cit., 107 ss.

${ }^{2}$ Cfr. REDLINK, L'ONU e «i signori della pace», cit., 70.
} 
sociali ${ }^{3}$.

Le contraddizioni esplose a seguito della fine del "bipolarismo" erano già presenti, pertanto, durante la "guerra fredda", in concomitanza con la seconda ondata della globalizzazione, laddove si ridefiniscono, rimanendo immutati, gli originari rapporti tra Paesi colonizzatori e colonizzati, fondati sulle logiche di sfruttamento e di dominio ${ }^{4}$ e sostanziantesi nel continuo esproprio delle risorse economiche da parte dei primi a danno dei secondi ${ }^{5}$; si registra, in tale fase, addirittura un aumento della disuguaglianza mondiale ${ }^{6}$. Funzionale a un'economia globalizzata è, allora, la rinuncia a un diritto internazionale quale possibile strumento di pace a vantaggio di un vero e proprio diritto di intervento definito "umanitario", attraverso il quale si ridefiniscono nuove forme di ingerenza "legalizzate".

Si affermano nuovi centri di potere politico privato che si vanno rapidamente sostituendo a quelli pubblici e che agevolano lo sviluppo di un costituzionalismo definito «multilevel», poiché volto all'accentuazione, anziché alla riduzione, dei differenti livelli di disuguaglianza espressione dell'eterogeneità dei singoli sistemi ordinamentali? .

Un esempio di istituto normativo strumentale ai processi di privatizzazione connotanti la società globale è offerto dalla Lex Mercatoria, che garantisce, nell'ambito degli affari transnazionali, la prevalenza del diritto privato $^{8}$.

La progressiva sostituzione della regolamentazione di natura privatistica in luogo di quella pubblicistica (fenomeno noto come constitution of private governance) è funzionale a quei processi di omologazione $\mathrm{e}$ standardizzazione espressione della terza ondata della globalizzazione economica9.

La crisi dello Stato costituzionale di diritto si manifesta, principalmente, attraverso il ricorso, da un lato, alla legislazione emergenziale e, dall'altro, alla guerra, che assume, a partire dall' 11 settembre 2001, i connotati della lotta al "terrorismo internazionale": attraverso entrambi i piani si dispiegano le strategie del controllo globale.

\section{LA MISTIFICAZIONE STATUNITENSE DELLA LOTTA AL TERRORISMO INTERNAZIONALE TRA POLITICHE DI INTERVENTO MILITARE NELLE AREE DI INTERESSE GEOPOLITICO E LEGISLAZIONE DELL'EMERGENZA}

Improntata all'unilateralismo è la strada intrapresa dagli Stati Uniti quale risposta agli attacchi alle Torri Gemelle di New York dell'11 settembre 2001.

Assistiamo, infatti, per quel che concerne la politica di intervento militare, a una definitiva delegittimazione

\footnotetext{
${ }^{3}$ Cfr. C. AMIRANTE, Union Européenne et libertés économiques entre marché, état et démocratie, in L'architecture du droit. Mélanges en l'honneur du Professeur Michel Troper, Economica, 2006, 35 ss.

${ }^{4}$ Ai fini di un approfondimento del "paradigma del dominio" e delle sue declinazioni, cfr. A.J. MAGLIACANE, Un Trauerspiel. L'intimazione di Riccardo III, in Costituzione, economia, globalizzazione. Liber Amicorum in onore di Carlo Amirante, Napoli, 2013, 399 ss.

${ }^{5} \mathrm{Cfr}$., a titolo esempilificativo, M. MANNO, L'ONU e la questione palestinese, in REDLINK (a cura di), L'ONU e «i signori della pace», cit., 117 ss.

${ }^{6}$ Cfr. J.R. CAPELLA, La globalizaciòn: ante una encrucijada politico-jurìdica, in Derecho y justicia en una sociedad global. Law and justice in a global society (Anales de la Càtedra Francisco Suàrez), Granada, España, Mayo 2005, 13 ss.

${ }^{7}$ Cfr. P. POLICASTRO, 'On The Reconstruction of the Legal Strength of the Constitution in a World in transition. Multi-Level Constitutionalism towards Multi-level Democracy', in J. NERGELIUS, P. POLICASTRO, K. URATA (a cura di), Challenges of Multilevel Constitutionalism, Proceedings of IVR $21^{\text {st }}$ World Congress, Lund 2003, Krakòw; P. POLICASTRO, A new Garment for an Old Question: 'A Clash between Man's Rights and Citizens' Rights in the Enlarged Europe', in J. NERGELIUS (a cura di), Nordic and other European Constitutional Traditions, Leiden/Boston, 2006, 66.

${ }^{8}$ C. AMIRANTE, Il futuro dell'Unione Europea dopo i referendum: tra economia, espertocrazia e democrazia, in S. GAMBINO, Trattato che adotta una Costituzione per l'Europa, Costituzioni nazionali, diritti fondamentali, Milano, 2006, 169 ss.

${ }^{9}$ Cfr. P. COLLIER, D. DOLLAR, Globalizzazione, crescita economica e povertà. Rapporto della Banca Mondiale, Bologna, 2003.
} 
del residuale ruolo svolto dal Consiglio di sicurezza dell'O.N.U. nell'autorizzare, quale non più esclusivo garante della pace, l'uso della forza militare, e, per quanto attiene all'ambito della produzione normativa, a un'ulteriore erosione delle già esigue garanzie affermate dal sistema giuridico interno e a un più generale disconoscimento dei diritti umani sanciti a livello universale (a esempio, dalla Convenzione di Ginevra del 1949).

Nel procedere in questa strategia interventista improntata all'unilateralismo, gli Stati Uniti disconoscono qualsiasi Organizzazione che possa imporre vincoli ai propri obiettivi, votando, a esempio, contro lo Statuto della Corte penale internazionale, adottato dalla Conferenza di Roma il 17 luglio 1998 ed entrato in vigore il $1^{\circ}$ luglio 2002, allo scopo di sottrarne al giudizio i propri crimini e criminali di guerra ${ }^{10}$.

Già l'intervento in Afghanistan, intrapreso il 7 ottobre 2001, in risposta agli attacchi alle Torri Gemelle di New York, cela, in realtà, interessi geopolitici statunitensi e non è mai stato esplicitamente autorizzato dal Consiglio di sicurezza dell'O.N.U., che, nella Risoluzione n. 1368, adottata il 12 settembre 2001, «dopo aver invocato l'impegno di tutti gli Stati a cooperare con urgenza per tradurre davanti alla giustizia esecutori e mandanti delle stragi», si limita a dichiarare, in maniera generica, «la sua pronta ed ampia disponibilità ad assumere tutte le iniziative necessarie in base alla Carta delle Nazioni Unite», con riferimento al capitolo VII della Carta medesima, «quello che regola l'esercizio del monopolio dell'uso della forza per il "mantenimento ed il ristabilimento della pace e della sicurezza internazionale" $\gg "$, e, nella successiva Risoluzione n. 1373, adottata il 28 settembre 2001, auspica un solerte impegno dei singoli Paesi nella repressione interna del terrorismo e il perfezionamento della cooperazione giudiziaria.

Le ragioni di una politica di aggressione militare come reazione all'11 settembre 2001, se già risultavano insufficienti in occasione dell'operazione «Enduring Freedom» in Afghanistan, sono ancor più inadeguate a giustificare la successiva invasione dell'Iraq, avviata nel marzo del 2003.

Nessuna delle tre Risoluzioni dell'O.N.U., menzionate dal governo statunitense quali fondamenti legittimanti la seconda guerra del Golfo, contiene, infatti, un'esplicita autorizzazione al conflitto. La stessa Risoluzione n. 1441, adottata nel novembre del 2002, si limita a stabilire che gli ispettori, inviati in Iraq per accertare l'esistenza di presunte armi di distruzione di massa, siano tenuti a «riferire» al Consiglio di sicurezza, il quale non ha, poi, comunque autorizzato l'uso della forza.

In ogni caso, attraverso il documento del 17 settembre 2002, gli Stati Uniti, strumentalizzando gli attacchi alle Torri Gemelle di New York, si riservano una «generale e illimitata possibilità [...] di tutelare i propri "interessi" nazionali con azioni non solo unilaterali, ma anche preventive, al fine soprattutto di difendersi dal terrorismo internazionale, e da altre forme moderne di aggressione» ${ }^{12}$.

Lo strumento della disinformazione strategica, posta in essere dai media occidentali, rappresenta un prezioso "alleato" delle strategie interventiste nordamericane ${ }^{13}$ (alla medesima stregua, la mistificazione mediatica ha agevolato e continua ad agevolare, nel passato come nel presente, i continui golpe, realizzati o anche solo tentati, ai danni dei governi "non allineati" in America Latina, la cui organizzazione è riconducibile agli U.S.A.).

Assistiamo, così, al definitivo affermarsi del concetto di «guerra preventiva» e «permanente», che caratterizzerà la politica statunitense di aggressione militare anche nel corso della successiva amministrazione Obama, al fine di continuare a legittimare interventi in aree di interesse geopolitico.

Le rivolte che, agli inizi del 2011, si sono estese dal Maghreb, sino a coinvolgere l'intera area del Mediterraneo, vanno a definire quelle nuove stagioni di protesta, conosciute come "Primavere Arabe", rispetto alle quali U.S.A. e U.E. si sono relazionati all'esclusivo scopo di estendere il proprio dominio su territori sino ad allora sottratti al proprio controllo.

\footnotetext{
${ }^{10}$ Cfr. M. MANDEL, Guerre illegali, danni collaterali e crimini contro l'umanità: il ruolo della legge contro i crimini internazionali dal Kosovo all'Iraq ed oltre, in Crit. dir., n. 1-2-3, 2004, 22.

${ }^{11} \mathrm{Cfr}$. V. MONETTI, La legislazione antiterrorismo dopo l'11 settembre: il contesto internazionale e l'Italia, in Ques. giust., n. 1, 2002, 51.

${ }^{12}$ Cfr. P. PICONE, La guerra contro l'Iraq e le degenerazioni dell'unilateralismo, in Rivista di diritto internazionale, 2003, I, 347.

${ }^{13}$ Cfr. M. TORREALTA (a cura di), Guerra e informazione. Un'analisi fuori da ogni schieramento, Sperling \& Kupfer, 2005.
} 
L'offensiva militare posta in essere contro la Libia di Gheddafi e il suo feroce assassinio hanno lasciato il Paese in una situazione di ingovernabilità, tale da consentire ogni genere di atrocità, perpetrate soprattutto nei confronti di migranti di altre etnie africane da parte delle milizie anti-Gheddafi.

La nuova fase della «guerra globale permanente» è scandita dal passaggio dall'unilateralismo statunitense all'intervento collettivo umanitario, fondato su una presunta «responsabilità di protezione», rinnovata fonte di legittimazione delle forme di aggressione militare di inizio decennio, di cui le vicende libiche offrono un esempio $^{14}$.

La destabilizzazione dell'Iraq, conseguenza della Seconda Guerra del Golfo, al pari delle interferenze statunitensi nel conflitto civile in Siria, rappresentano due delle principali cause dell'attuale crisi mediorientale. Operazioni di Intelligence nordamericana, israeliana e dei partner occidentali (compresa l'Italia) hanno avuto a oggetto l'addestramento di guerriglieri sunniti, al fine di organizzare attacchi contro il premier siriano Bashar al-Assad. Siffatti mercenari sono stati, quindi, reclutati dall'ISIS e la situazione è sfuggita al controllo degli Stati Uniti e dei loro alleati.

L'offensiva dello Stato Islamico contro le popolazioni al confine tra Iraq e Siria è l'effetto, pertanto, di interventi militari finalizzati alla destabilizzazione di Paesi Arabi non "allineati" agli U.S.A., come la Siria di Bashar al-Assad.

Nella Risoluzione n. 2170, adottata il 15 agosto 2014, il Consiglio di Sicurezza delle Nazioni Unite deplora e condanna, tuttavia, gli atti terroristici dell'ISIS, la violenta ideologia estremista e le continue violazioni dei diritti umani e del diritto internazionale umanitario.

Attraverso la successiva Risoluzione n. 2178, adottata all'unanimità (15 voti a zero) il 24 settembre 2014, il Consiglio medesimo invita gli Stati Membri a prevenire e contrastare i fenomeni di reclutamento di individui finalizzato al compimento di atti terroristici.

Nonostante le condanne da parte delle Nazioni Unite e, in generale, della comunità internazionale, una vera e propria guerra di resistenza all'ISIS, a partire dalla liberazione della città siriana di Kobane, in prossimità del confine turco, è stata intrapresa, nel 2014, dal solo popolo e dalle organizzazioni curde.

Solo in una fase successiva possiamo parlare di un intervento sinergico di realtà non omogenee - nell'ambito delle quali anche la potenza nordamericana, sia pur mossa dall'esclusivo interesse di pianificare nuove strategie di attacco alla Siria, ha preso parte - sino alla riconquista di Raqqa, capitale dello Stato Islamico, avviata alla fine di settembre 2017.

L'altro livello attraverso il quale si estrinseca la reazione degli U.S.A. agli attacchi alle Torri Gemelle di New York è la legislazione dell'emergenza, che, frutto di una "cultura" fortemente radicata nei moderni ordinamenti statuali, si impone attraverso l'alterazione della fonte di legittimazione giuridica, trovando, quindi, una stabile collocazione all'interno di un sistema, quello statunitense, di cui compromette le già esigue garanzie costituzionali, con gravi ripercussioni sulla sfera di libertà dei singoli, cittadini o stranieri presenti sul territorio.

Misure di carattere emergenziale saranno adottate dagli Stati Uniti all'indomani degli attacchi dell'11 settembre. Le tre principali tappe di siffatta legislazione, attraverso la quale la libertà e i diritti fondamentali dell'individuo sono sacrificate sull'altare della "sicurezza nazionale", sono rappresentate dall'approvazione, da parte del Senato, dell'Usa Patriot Act, il 26 ottobre 2001, del President Issues Military Order, il 13 novembre 2001 e del Military Commission Act, firmato da George W. Bush il 17 ottobre 2006, al fine di detenere all'infinito soggetti ritenuti arbitrariamente, dal governo statunitense, presunti terroristi.

Segnali in una direzione maggiormente garantista, anche se destinati a rappresentare episodi isolati, sono provenuti, a seguito dei due provvedimenti del 2001, da alcune pronunce della Corte Suprema U.S.A. in ordine ad habeas corpus petitions, presentate nell'interesse di alcuni prigionieri di Guantanamo (tra questi, particolare attenzione è stata dedicata ai ricorsi a favore di due cittadini americani, Hamdi e Padilla, il secondo dei quali arrestato addirittura a Chicago, all'interno dei confini nazionali, in territorio, quindi,

\footnotetext{
${ }^{14}$ Cfr. M. MANDEL, R2P \& ICC v. UNC: The Responsibility to Protect and the International Criminal Court versus the Charter of the United Nations, in Costituzione, economia, globalizzazione. Liber Amicorum in onore di Carlo Amirante, cit., 1375 ss.
} 
estraneo alle zone attive di combattimento) $)^{15}$.

Nella sentenza Hamdi v. Rumsfeld del 28 giugno 2004, la Corte Suprema ebbe a precisare che «la detenzione di un cittadino non può essere eseguita al di fuori del principio costituzionale del due process, ovvero, nel caso specifico, del diritto del detenuto di contestare quella qualificazione arbitrariamente attribuita dal Governo, conoscere gli elementi di fatto base di tale status e contrastarli di fronte ad un "neutral decision maker" ${ }^{16}$; in analoga direzione si è mossa la U.S. Court of Appeals for the second circuit di New York, attraverso la sentenza del 18 dicembre 2003, avente a oggetto l'habeas corpus appeal presentato a favore di Jose Padilla, la cui decisione ha trovato, poi, conferma nel successivo intervento della Corte Suprema (Rumsfeld v. Padilla, del 28 giugno 2004).

Le pronunce in esame, pur rappresentando una dovuta presa d'atto di palesi irregolarità, sono riferite a singole vicende eclatanti, aventi a oggetto cittadini americani e non rappresentano una piena condanna dell'operato dell'amministrazione Bush, la cui facoltà di detenere in via indefinita gli «unlawful combatans» non viene, comunque, contestata in assoluto, ma subordinata all'autorizzazione del Congresso.

Si è parlato, al riguardo, di sentenze «meritorie», o di «non decisioni», volte a riaffermare un teorico dovere dell'esecutivo al rispetto dei principi costituzionali, fornendo, tuttavia, al medesimo ampi spazi di agibilità per l'aggiramento, nella prassi, di tali principi ${ }^{17}$.

Nella prima decade del nuovo millennio la prigione lager di Guantanamo assurge a emblema della violazione dei diritti umani ${ }^{18}$. Sarebbe, allora, superfluo interrogarsi se - in uno spazio detentivo sottratto, da un lato, al controllo giudisdizionale e, dall'altro, alle garanzie contemplate dalle quattro Convenzioni di Ginevra del 1949 (e, quindi, a ogni forma di tutela), nel cui perimetro i soggetti presenti sono sequestrati a tempo indeterminato, sine die e sine iudicio, e sottoposti a tortura - sia assicurato il rispetto delle differenze di ordine sociale, culturale e religioso esistenti tra i detenuti. I diritti dei prigionieri islamici non possono certo acquisire cittadinanza in un luogo siffatto ${ }^{19}$. La strategia del terrore posta in essere da Osama Bin Laden $^{20}$ - alla medesima stregua di quanto accade adesso in relazione a quella pianificata dal Califfo alBaghdadi - è strumentalizzata al fine di dispiegare un controllo sulla totalità degli individui, sia sui cittadini statunitensi considerati arbitrariamente presunti terroristi, sia su tutte le altre popolazioni, soprattutto quelle che abitano i territori sui quali insistono gli interessi nordamericani.

Gli attacchi dell'11 settembre 2001, se sul piano esterno diventano la fonte di legittimazione per la «guerra preventiva», sul piano interno offrono, allora, l'occasione al governo degli Stati Uniti per smantellare le già esigue garanzie costituzionali. Ampliando a dismisura le funzioni dell'esecutivo e minando, di conseguenza, il meccanismo del «checks and balances», espressione della separazione dei poteri, si dispone, infatti, di maggiori strumenti repressivi, utilizzati prevalentemente nei confronti di oppositori politici e migranti, cui vengono estese più invasive forme di controllo. Le attuali politiche dell'esclusione poste in essere, a partire dal 2017, dall'Amministrazione Trump - che si spingono sino ad abrogare il Deferred Action for Childhood Arrivals, il programma di amnistia per i soggetti minori di età fatti entrare illegalmente negli Stati Uniti, previsto da un provvedimento adottato dalla precedente Amministrazione Obama nel 2012 - sono il risultato del ciclico riannodarsi di antiche trame xenofobe connotanti la tradizione delle classi dominanti (in primis, repubblicane) nordamericane.

\section{I PROVVEDIMENTI COMUNITARI ISPIRATORI DELLA PRIMA LEGISLAZIONE ANTITERRORISTICA ITALIANA}

\footnotetext{
${ }^{15}$ Cfr. M. MIRAGLIA, Paura e libertà (Legislazione antiterrorismo e diritti di difesa negli Stati Uniti), in Ques. giust., n. 2-3, 2004, 304 ss.

${ }^{16}$ Cfr. M. MIRAGLIA, Lotta al terrorismo e diritti dei prigionieri: la Corte Suprema U.S.A. richiama al rispetto dei principi costituzionali, in Dir. pen. e proc., n. 11, 2004, 1426.

${ }^{17}$ Cfr. M. MIRAGLIA, Una nuova normalità: metamorfosi della giustizia penale statunitense dopo l'11 settembre, in Cass. pen., n. 9, 2005, 2832-2834.

${ }^{18}$ Cfr. M. RATNER, E. RAY, Prigionieri di Guantanamo. Quello che il mondo deve sapere, Bologna, 2005.

${ }^{19}$ Cfr. A. STARACE, I diritti dei prigionieri islamici, in Innovazione e diritto, n. 4, 2007, 80 ss.

${ }^{20}$ Cfr. P.L. BERGEN, Holy war, Inc. Osama bin Laden e la multinazionale del terrore, Milano, 2001.
} 
Il posizionamento dell'U.E. al fianco degli Stati Uniti nella lotta al c.d. "terrorismo internazionale", all'indomani degli attacchi alle Torri Gemelle di New York, è stato immediato.

Attraverso la «Proposta di decisione-quadro» ${ }^{21}$, del 19 settembre 2001, e le «Conclusioni della Presidenza e Piano di Azione» ${ }^{22}$, del 21 settembre del medesimo anno, Commissione Europea e Consiglio Europeo hanno proposto, a livello embrionale, una definizione di atti terroristici cui si sarebbero dovuti uniformare gli Stati membri dell'U.E.

La «proposta» darà luogo alla decisione quadro 2002/475/GAI, sulla «lotta contro il terrorismo», adottata il 13 giugno 2002 dal Consiglio Giustizia e Affari interni. Al concetto di reato terroristico, definito dal provvedimento comunitario, si adeguerà il legislatore italiano nel definire la propria disciplina penale in materia.

Il 13 giugno 2002 viene, altresì, adottata la decisione quadro 2002/584/GAI, avente a oggetto il mandato d'arresto europeo, che si pone anch'essa quale obiettivo principale la creazione di una cooperazione penale tra gli Stati membri, consistente nel reciproco automatico riconoscimento tra le rispettive autorità giudiziarie, vincolate da un sistema di consegna fondato su un mandato di cattura europeo, che sostituisce, pertanto, le tradizionali procedure di estradizione ${ }^{23}$.

Ancora una volta il superamento del concetto di «Stato-nazione» viene invocato al fine di legittimare interventi in campo repressivo, laddove si determina un'unità nella "lotta al nemico", che diventa, così, "interno" ai singoli Paesi.

Alla «cooperazione di polizia e giudiziaria in materia penale» è, altresì, riconducibile il Piano di Azione, approvato dal Consiglio Europeo il 21 settembre 2001, a seguito di una riunione straordinaria tenutasi all'indomani degli attacchi dell'11 settembre.

Nella prima parte delle Conclusioni della Presidenza, si esprime piena solidarietà agli U.S.A. e si ribadisce la propria fermezza nella lotta al "terrorismo internazionale", assunta quale obiettivo prioritario dall'Unione; si riafferma, in particolare, la legittimità della reazione statunitense sulla scorta della Risoluzione n. 1368, adottata dal Consiglio di sicurezza dell'O.N.U. il 12 settembre 2001, e si auspica la costituzione di una coalizione globale antiterroristica sotto l'egida delle Nazioni Unite, allargata anche alla Federazione russa, ai partner arabi e musulmani e a qualsiasi altro Paese disposto alla difesa dei «valori comuni» ${ }^{24}$.

Nella successiva parte dedicata alla politica comunitaria di lotta al terrorismo, il Consiglio approva il Piano di Azione, che si articola in sette punti, i cui primi tre hanno per obiettivo il rafforzamento della cooperazione giudiziaria e di polizia. Nel primo di questi si afferma la necessità di istituire l'ordine di arresto europeo, che possa consentire «la consegna diretta delle persone ricercate da autorità giudiziaria ad autorità giudiziaria, garantendo al tempo stesso i diritti e le libertà fondamentali».

Attraverso il secondo punto, il Consiglio europeo «chiede al Consiglio "Giustizia e affari interni" di far procedere all'identificazione dei presunti terroristi in Europa nonché delle organizzazioni che li sostengono per compilare un elenco comune delle organizzazioni terroristiche», invitando, al riguardo, a «una migliore cooperazione e un migliore scambio» di notizie «tra tutti i servizi di informazione dell'Unione», al cui fine «saranno istituite squadre investigative comuni».

E, ai sensi del terzo punto, «gli Stati membri scambieranno senza indugio e sistematicamente con l'Europol tutti i dati utili in materia di terrorismo», con l'auspicio che tra Europol e competenti autorità statunitensi si possa concludere un accordo di cooperazione entro la fine dell'anno.

I successivi obiettivi che il Consiglio europeo si pone, ai punti quarto e quinto, sono, rispettivamente,

${ }^{21}$ Cfr. Commissione europea, Proposta di decisione-quadro sulla lotta contro il terrorismo, Bruxelles, 19 settembre 2001, in Rivista Italiana di Diritto Pubblico Comunitario, 2001, n. 5, 883 ss., con nota di M. BONINI, La «lotta» al terrorismo: il quadro giuridico internazionale e comunitario.

${ }^{22}$ Cfr. Consiglio europeo straordinario, Conclusioni della Presidenza e Piano di Azione, Bruxelles, 21 settembre 2001, in Rivista Italiana di Diritto Pubblico Comunitario, 2001, n. 5, 895 ss., con nota di M. BONINI, Oltre lo «Statonazione» per una politica europea di «lotta» al terrorismo.

${ }^{23}$ Cfr. N. BARTONE, Mandato di arresto europeo e tipicità nazionale del reato, Milano, 2003, con prefazione di G. VASSALLI, 296.

${ }^{24}$ Cfr. Consiglio europeo straordinario, Conclusioni della Presidenza e Piano di Azione, Bruxelles, 21 settembre 2001 , cit., 895-896. 
l'attuazione di tutte le convenzioni in materia, al fine di un rapido sviluppo degli strumenti giuridici internazionali, e la predisposizione di misure di contrasto al finanziamento di gruppi terroristici.

Il Piano di Azione prevede, infine, il rafforzamento della sicurezza aerea e il coordinamento dell'azione globale dell'U.E., affidata al Consiglio Affari generali.

Nelle Conclusioni della Presidenza si rinnovano, quindi, le speranze che «l'Unione partecipi più attivamente agli sforzi della Comunità internazionale per prevenire e stabilizzare i conflitti regionali», sviluppando «la politica estera e di sicurezza comune (PESC) e rendendo al più presto operativa la politica europea di sicurezza e di difesa (PESD) $\rangle^{25}$.

Il superamento del concetto di «Stato-nazione» sarebbe, allora, auspicabile, in vista dei più generali interessi comunitari, innanzitutto in campo giuridico ${ }^{26}$.

Per assecondare le esigenze espresse nel punto quinto del Piano di Azione, il Consiglio Affari generali ha adottato, il 27 dicembre 2001, una posizione comune, la n. 2001/931/PESC, relativa all'applicazione di misure specifiche per la lotta al terrorismo, aventi a oggetto persone, gruppi o entità indicate in un'apposita lista allegata al provvedimento in esame e nei cui confronti verrà ordinato il congelamento di capitali, risorse finanziarie o economiche e servizi finanziari ${ }^{27}$.

La decisione quadro 2002/475/GAI, che rappresenta le fondamenta della politica antiterroristica dell'U.E., è stata successivamente modificata dalla decisione quadro 2008/919/GAI, adottata il 28 novembre 2008.

È da sottolineare, infine, come, nel corso del primo quindicennio del nuovo millennio, operazioni di intelligence abbiano parallelamente affiancato - sia livello comunitario, sia a livello sovranazionale - $\mathrm{i}$ provvedimenti legislativi nel contrasto al terrorismo, legittimando limitazioni alla libertà personale (sino a procedere a veri e propri sequestri di persona, le cosiddette "extraordinary renditions" o "consegne straordinarie" 28 , e a rendere sistematica l'applicazione del metodo della tortura) e compressioni al diritto alla privacy degli individui. In relazione a tale ultimo aspetto, all'indomani dei primi attacchi a Parigi del 7 gennaio 2015, si rilancia la tematica della creazione di un «sistema comune per la raccolta e lo scambio tra gli Stati membri dei dati riguardanti i passeggeri del traffico aereo (PNR, Passenger Name Records)» ${ }^{29}$. Nella nuova fase inaugurata dagli attacchi alla rivista satirica francese Charlie Hebdo, uno degli obiettivi dell'intelligence europea è rappresentata dal rendere tracciabili gli spostamenti e di poter, così, ricostruire le abitudini dei viaggiatori sospetti, soprattutto dei possibili inviati in campi di addestramento mediorientali, implementando le «attività di memorizzazione e analisi di determinati supporti conoscitivi, nella prospettiva di attuare una metodologia "combinata" di law enforcement ${ }^{30}$, al fine di rafforzare la prevenzione del c.d. "terrorismo islamico".

\section{MISURE DI CONTRASTO AL TERRORISMO INTERNAZIONALE NELLA LEGISLAZIONE PENALE ITALIANA}

Il percorso di adeguamento della disciplina antiterroristica italiana alle indicazioni europee si articola attraverso tre principali tappe, rappresentate da altrettanti interventi legislativi, adottati a seguito di attentati

\footnotetext{
${ }^{25}$ Cfr. Consiglio europeo straordinario, Conclusioni della Presidenza e Piano di Azione, Bruxelles, 21 settembre 2001, cit., 897-898.

${ }^{26}$ Cfr. M. BONINI, Oltre lo «Stato-nazione» per una politica europea di «lotta» al terrorismo, cit., 901.

${ }^{27}$ Il summenzionato Piano d'azione del 2001 è riveduto dal Consiglio europeo, che, in data 15 giugno 2004, adotta un nuovo Piano, il quale, tenendo conto degli obiettivi approvati dal Consiglio medesimo il 25 marzo 2004, allindomani degli attentati di Madrid, l'11 marzo 2004, concretizza nuove strategie nella lotta al terrorismo internazionale, prevedendo scadenze per il raggiungimento di progressi determinati in settori specifici.

${ }^{28}$ Cfr., sul punto, D. RUSSO, Lotta al terrorismo internazionale e diritti umani: le "extraordinary renditions" nella giurisprudenza di Strasburgo, in Legisl. pen., n. 2, 2013, 319 ss.

${ }^{29}$ Cfr. B. PIATTOLI, Principio di proporzionalità UE e trattamento dei dati personali nella lotta al terrorismo, in Dir. pen. e proc., n. 7, 2015, 885 ss.

${ }^{30}$ Cfr. B. PIATTOLI, Principio di proporzionalità UE e trattamento dei dati personali nella lotta al terrorismo, cit., 885.
} 
epocali ${ }^{31}$.

All'indomani degli attacchi alle Torri Gemelle di New York, il legislatore ebbe a recepire l'internazionalità della finalità terroristica, attraverso la modifica dell'art. 270bis c.p. La repressione del fenomeno del terrorismo internazionale avviene, in primo luogo, con l'ausilio dello strumento associativo, a opera del D.L. 18 ottobre 2001, n. 374, convertito, con modificazioni, nella L. 15 dicembre 2001, n. $438^{32}$.

L'introduzione del comma 3 nell'art. 270bis c.p. estende la finalità terroristica anche agli atti di violenza rivolti contro uno Stato estero, un'istituzione o un organismo internazionale. Il legislatore del 2001 non fornisce, tuttavia, una definizione di terrorismo.

La reazione agli attacchi alla metropolitana di Londra, il 7 luglio 2005, è rappresentata dal D.L. 27 luglio 2005, n. 144 (c.d. «decreto Pisanu»), convertito nella L. 31 luglio 2005, n. 155. Il legislatore del 2005, nel configurare l'art. 270sexies c.p., mutua la definizione di atti terroristici contenuta nella decisione quadro 2002/475/GAI. Saranno punite, allora, anche alla luce del sistema penale italiano, gli atti intenzionali che, «per la loro natura o contesto, possono arrecare grave danno a un paese o a un'organizzazione internazionale, quando sono commessi al fine di»: «intimidire gravemente la popolazione», ovvero «costringere indebitamente i poteri pubblici o un'organizzazione internazionale a compiere o astenersi dal compiere un qualsiasi atto» o ancora «destabilizzare gravemente o distruggere le strutture politiche fondamentali, costituzionali, economiche o sociali di un paese o un'organizzazione internazionale».

Gli attentati di Londra forniscono, inoltre, al legislatore italiano l'occasione per rendere penalmente rilevanti, attraverso gli artt. 270quater e 270 quinquies c.p., le condotte, rispettivamente, di arruolamento e addestramento di "combattenti islamici", optando, in tal modo, per un'esplicita scelta di campo in relazione alle politiche statunitensi di aggressione militare in Medio Oriente.

Alla luce della L. n. 155/2005 acquistano rilevanza penale le figure, da un lato, del solo arruolatore, e non anche quella dell'arruolato (art. 270quater c.p.), poiché soggetto ancora non pericoloso, e, dall'altro, quelle sia dell'addestratore sia dell'addestrato (art. 270quinquies c.p.); la pericolosità di quest'ultimo è ricollegata alla circostanza che egli abbia già acquisito gli strumenti necessari per la realizzazione di attività terroristiche.

Gli attentati alla rivista satirica francese Charlie Hebdo hanno indotto l'esecutivo ad adottare il D.L. 18 febbraio 2015, n. 7, convertito, con modificazioni, nella L. 17 aprile 2015, n. 43. Attraverso siffatto intervento legislativo sono apportate, innanzitutto, modifiche agli artt. 270quater e 270quinquies c.p., precedentemente introdotti dalla L. 31 luglio 2005, n. 155, al fine di ampliare il novero delle figure delittuose ivi previste ${ }^{33}$.

L'offensiva dello Stato Islamico, per un verso militare, per l'altro mediatico, è ascrivibile alla terza fase della jihad in Europa ${ }^{34}$ : l'utilizzo della rete, da un lato, si va a sostituire ai luoghi fisici (scuole, istituti culturali o moschee) ai fini dell'attività di reclutamento e, dall'altro, pone a disposizione degli utenti il know how per l'addestramento finalizzato ad atti terroristici. Destinatari dell'invito jihadista - teso a creare suggestioni nei confronti di vittime di discriminazioni, sia musulmani di seconda o terza generazione, di nascita o residenza europea, sia di cittadini comunitari convertiti all'islamismo - sono soggetti sovente marginalizzati, che intravedono nelle promesse del Califfato un'alternativa alle società dell'esclusione ${ }^{35}$.

Lo strumento telematico e i social network attribuiscono, da un lato, un ruolo attivo all'arruolando nella ricerca di una formazione jihadista di riferimento e consegnano, dall'altro, a chi intenda intraprendere percorsi individuali di auto-addestramento, agevoli strumenti per l'apprendimento di tecniche finalizzate ad

\footnotetext{
${ }^{31}$ Ai fini di una più esaustiva disamina delle tre principali tappe attraverso le quali si articola la legislazione italiana di contrasto al terrorismo internazionale, cfr. U. NAZZARO, Le misure di contrasto al terrorismo internazionale alla luce della Legge 17 aprile 2015, n. 43, in Riv. pen., n. 10, 2015, 822 ss.

${ }^{32}$ Per un approfondimento degli aspetti penali della normativa antiterroristica italiana, cfr. U. NAZZARO, Il diritto penale del nemico tra delitto di associazione politica e misure di contrasto al terrorismo internazionale, Giannini (Quaderni dell'Accademia Pontaniana, n. 62), Napoli, 2016.

${ }^{33}$ Cfr. S. LICCIARDELLO, Nuove norme antiterrorismo in Italia, in www.sicurezzanazionale.gov.it., 9 settembre 2016.

${ }^{34}$ Cfr. F. MARONE, Il nuovo volto del terrorismo jihadista, in La rivista il Mulino, 2, 2015, 293 ss.

${ }^{35} \mathrm{Cfr}$. M. FLORES, Le domande che non ci poniamo, in La rivista il Mulino, cit., 318 ss.
} 
attività terroristiche.

L'arruolato, che manifesti il proprio intento di raggiungere campi di addestramento paramilitari in Medio Oriente, e l'auto-addestrato, che prescinda dall'instaurazione di un rapporto di tipo militare con l'addestratore per la propria formazione, rappresentano due nuove figure penalmente rilevanti introdotte dal legislatore del 2015, contemplate attraverso l'ampliamento, rispettivamente, degli artt. 270quater e $270 q u i n q u i e s$ c.p.

La Corte di Cassazione, con sentenza n. 40699, depositata il 9 ottobre 2015, sottolinea, in relazione alla nozione di arruolamento, il carattere della serietà dell'accordo, «intesa da un lato come autorevolezza della proposta (il proponente deve avere la concreta possibilità di inserire l'aspirante nella struttura operativa una volta concluso l'ingaggio) e dall'altro come fermezza della volontà di adesione al progetto».

Attraverso la L. 17 aprile 2015, n. 43, viene, altresì, introdotto l'art. 270quater. 1, ai sensi del quale è previsto il reato di organizzazione di trasferimenti per finalità di terrorismo. A integrare le fattispecie delittuose ivi previste intervengono tre nuove figure penalmente rilevanti: il finanziatore, l'organizzatore e colui che propaganda viaggi in territorio estero finalizzati al compimento di attività terroristiche.

La sentenza n. 8 della Corte d'Assise di Milano, depositata il 24 febbraio 2017, rappresenta la prima condanna per il reato di cui all'art. 270quater.1: nella pronuncia si stigmatizza che l'espressione "trasferimenti", declinata al plurale, abbia l'esclusiva «funzione di ricomprendere sotto un'unica disposizione sia l'ipotesi in cui venga posta in essere una singola condotta, sia l'ipotesi in cui vengano poste in essere più condotte dello stesso tipo in un unico contesto temporale ${ }^{36}$. Assume, quindi, rilevanza penale, alla luce della decisione in esame, l'attività dell'organizzare anche un solo viaggio in territorio estero per il compimento di attività terroristiche, ma non l'auto-organizzazione di quello proprio.

L'obiettivo del legislatore del 2015 è quello di contrastare due aspetti di un medesimo fenomeno: i "foreign fighters", ossia i combattenti stranieri che prendono parte ai conflitti in Medio Oriente o in Nord Africa, e i "lupi solitari", che, agendo autonomamente e rivendicando, nel contempo, la propria appartenenza all'ISIS, trasformano le città europee o i luoghi ove è rilevante la presenza di cittadini occidentali in nuovi teatri di guerra.

Perplessità sussistono, tuttavia, in relazione alla scelta di arginare i nefasti effetti delle politiche statunitensi ed europee di ingerenza a livello globale - nel caso specifico in Medio Oriente e Nord Africa, generatrici, nell'ipotesi in esame, dell'offensiva militare del Califfato, che dalle regioni mediorientali e nordafricane è esportata in Occidente - attraverso lo strumento della legislazione emergenziale antiterroristica: siffatte misure attinenti al diritto, se da un lato mostrano la propria inadeguatezza ad arginare azioni inquadrabili in veri e propri conflitti bellici, sono, dall'altro, esclusivamente idonee alla mera repressione e al controllo degli individui, che subiscono, in generale, gravi limitazioni al diritto alla privacy e alla libertà personale. Paradossali vittime di un'artefatta opinione pubblica e dei conseguenti interventi legislativi sono, sovente, proprio i migranti e i rifugiati, in fuga dagli orrori di guerre intraprese dalle medesime potenze occidentali. Gli incerti confini delle figure penalmente rilevanti, introdotte progressivamente dal legislatore italiano attraverso le successive tappe che segnano il suo intervento in materia, attribuiscono al giudice ampi margini di discrezionalità nell'applicazione delle norme.

Diventa agevole, così, innanzi a un fenomeno, quello dell'offensiva jihadista, definito di «terrorismo individuale» - poiché improntato a un «modello orizzontale», ove, anziché innanzi a una struttura verticistica gerarchicamente organizzata, ci troviamo di fronte a una fitta rete di cellule funzionalmente collegate - ricondurre nell'alveo dell'organizzazione un ventaglio sempre più ampio di ipotesi. A tal proposito, la Corte di Assise di Milano ${ }^{37}$, con sentenza depositata il 28 luglio 2016, ravvisa nelle «"condotte univocamente sintomatiche, consistenti nello svolgimento di attività preparatorie rispetto alla esecuzione

\footnotetext{
${ }^{36}$ D. ALBANESE, Le motivazioni della Corte d'Assise di Milano sul "caso Fatima": spunti di riflessione su terrorismo internazionale e organizzazione di trasferimenti ex art. 270-quater.1 c.p., in Dir. pen. cont., 28 marzo 2017, www.penalecontemporaneo.it.

${ }^{37}$ Cfr., a commento della sentenza, D. ALB ANESE, Partecipazione all'associazione con finalità di terrorismo 'Stato Islamico': una pronuncia di condanna della Corte d'Assise di Milano, in Dir. pen. cont., 21 ottobre 2016, www.penalecontemporaneo.it.
} 
del programma" la spia dell'effettivo inserimento degli imputati nell'associazione terroristica». L'agire dell'associato deve, tuttavia, palesare la peculiare finalità di terrorismo, dal momento che l'indagine sulla sussistenza o meno del sodalizio teso alla diffusione del terrore comporta «un percepibile spostamento del baricentro valutativo verso la componente psichica del delitto».

Le attività di istigazione pubblica - compiute, attraverso i social network, da parte di chi abbia giurato fedeltà al Califfato - in quanto dirette «a commettere atti di violenza con finalità terroristica nei confronti dei cittadini italiani», sono, pertanto, da considerare riconducibili, ad avviso della Corte di Assise di Milano, nell'alveo dell'art. 270bis c.p.

Il mero proselitismo o indottrinamento al martirio non è stato, viceversa, ritenuto sufficiente a integrare - ai sensi della pronuncia n. 48001 della Corte di Cassazione, depositata il 14 novembre 2016 - la fattispecie delittuosa in esame ${ }^{38}$.

A completare le misure di contrasto al terrorismo internazionale interviene il legislatore del 2016, al fine di procedere alla ratifica ed esecuzione di una serie di convenzioni adottate a livello europeo o internazionale. Attraverso la L. 28 luglio 2016, n. $153^{39}$, alle norme del codice penale italiano in materia sono affiancati gli ulteriori artt. 270quinquies.1, 270quinquies.2 e 280ter, che adeguano la legislazione antiterroristica italiana a quella comunitaria e sovranazionale ${ }^{40}$.

Ai sensi dell'art. 270quinquies.1 c.p., è punito con la reclusione da sette a quindici anni chiunque, al di fuori dei casi di cui agli articoli 270bis e 270quater.1, raccoglie, eroga o mette a disposizione beni o denaro, in qualunque modo realizzati, destinati a essere in tutto o in parte utilizzati per il compimento delle condotte con finalità di terrorismo di cui all'art. 270sexies c.p., indipendentemente dall'effettivo utilizzo dei fondi reperiti; è, altresì, disposta la detenzione da cinque a dieci anni nei confronti di chi deposita o custodisce le summenzionate risorse economiche.

Assume, così, rilevanza penale, allo scopo di adeguare l'ordinamento italiano ai dettami europei e sovranazionali, il finanziamento di attività tese alla diffusione del terrore. Si tratta di una fattispecie delittuosa residuale, integrata da condotte non inquadrabili nel modello associativo, né aventi a oggetto l'organizzazione di viaggi in territorio estero finalizzati alla realizzazione di atti di terrorismo. Il finanziatore, infatti, non deve, da un lato, essere riconducibile al sodalizio criminoso di cui all'art. 270bis c.p. e il suo agire deve esulare, dall'altro, dal mero finanziamento di trasferimenti tesi al compimento di attività terroristiche.

Viene, quindi, punito, ai sensi del successivo art. 270quinquies.2 c.p., con la reclusione da due a sei anni e con la multa da euro tremila a quindicimila chiunque sottrae, distrugge, disperde, sopprime o deteriora beni o denaro, sottoposti a sequestro per prevenire il finanziamento delle condotte con le finalità di cui all'art. 270 sexies c.p.

A completare il ventaglio delle nuove ipotesi incriminatrici interviene, a punire gli atti di terrorismo nucleare, l'art. 280 bis c.p. È prevista la reclusione non inferiore ai quindici anni nei confronti di chi, per il perseguimento delle finalità di cui all'art. 270sexies c.p., procura a sé o ad altri materia radioattiva o crea un ordigno nucleare ovvero ne viene altrimenti in possesso. L'utilizzo del sopra citato materiale o di un impianto nucleare ovvero il danneggiamento di quest'ultimo, che possa comportare anche il solo pericolo della diffusione della radioattività, è sanzionato con la detenzione non inferiore a venti anni. Le pene contemplate nei primi due commi dell'art. 280bis c.p. si applicano, altresì, quando le summenzionate

\footnotetext{
${ }^{38}$ Cfr. R. BERTOLESI, Indottrinare al martirio non è reato di associazione con finalità di terrorismo, in Dir. pen. cont., 23 gennaio 2017, www.penalecontemporaneo.it.

39 «Norme per il contrasto al terrorismo, nonché ratifica ed esecuzione: a) della Convenzione del Consiglio d'Europa per la prevenzione del terrorismo, fatta a Varsavia il 16 maggio 2005; b) della Convenzione internazionale per la soppressione di atti di terrorismo nucleare, fatta a New York il 14 settembre 2005; c) del Protocollo di Emendamento alla Convenzione europea per la repressione del terrorismo, fatto a Strasburgo il 15 maggio 2003; d) della Convenzione del Consiglio d'Europa sul riciclaggio, la ricerca, il sequestro e la confisca dei proventi di reato e sul finanziamento del terrorismo, fatta a Varsavia il 16 maggio 2005; e) del Protocollo addizionale alla Convenzione del Consiglio d'Europa per la prevenzione del terrorismo, fatto a Riga il 22 ottobre 2015».

${ }^{40} \mathrm{Cfr}$. F. POLINO, Il contrasto alle nuove forme di terrorismo internazionale, in www.magistraturaindipendente.it, 6 marzo 2017.
} 
condotte ivi descritte abbiano a oggetto materiali o aggressivi chimici o batteriologici.

\section{CONCLUSIONE}

L'esempio più drammatico della situazione di ingovernabilità, creata a seguito della destabilizzazione di aree di interesse geopolitico, diretta conseguenza delle strategie di aggressione militare statunitense ed europea, è offerto dal progressivo espandersi, a partire dal 2014, dell'autoproclamato Stato Islamico, dapprima nei territori compresi tra Iraq e Siria, ai confini con la Turchia, e, quindi, in Libia.

Nonostante le Risoluzioni ONU e le formali dichiarazioni dei premier di Stati Uniti e dei Paesi dell'U.E., il ruolo principale nella guerra all' ISIS è, sin dall'inizio del conflitto, svolto dalle organizzazioni curde - come il PKK, l'YPJ ${ }^{41}$ e l'YPG - già in passato inserite nelle "liste nere" dal governo turco. Appare paradossale, allora, come gruppi, ritenuti terroristici dagli attori istituzionali occidentali e dai loro partner strategici mediorientali, siano i principali oppositori al vero terrorismo, di cui il Califfato è la più immediata espressione.

Il governo di Erdogan ha posto in essere, soprattutto all'inizio del conflitto esploso nel 2014, azioni di disturbo nei confronti della resistenza curda, bombardando, a esempio, le postazioni militari del PKK nei pressi di Kobane, luogo simbolo della lotta di liberazione del Kurdistan dall'occupazione del Daesh, come viene in maniera dispregiativa definita l'organizzazione del Califfo al-Baghdadi.

Solo in una fase successiva, l'esplodere delle contraddizioni legate alle nefandezze poste in essere dallo Stato Islamico - perpetrate nei confronti delle popolazioni delle regioni mediorientali e africane, ma stigmatizzate solo in relazione agli attentati compiuti nelle metropoli europee (Parigi, Bruxelles o Barcellona) o nelle città ove rilevante è la presenza di turisti occidentali (Tunisi o Sousse) - ha imposto all'Amministrazione statunitense di prendere una chiara posizione circa la necessità di sconfiggere l'ISIS. La deposizione del governo di Bashar al-Assad continua, tuttavia, a rappresentare l'obiettivo principale nordamericano: il perseguire siffatte politiche di intromissione nei confronti dei Paesi "non allineati" (rectius, non subalterni) alle posizioni degli U.S.A. ha alimentato la tensione dei rapporti con la Russia, contraria alle ingerenze nei confronti della Siria, e ha reso impraticabile, per un eccessivo lasso di tempo, una comune, disinteressata ed efficace offensiva al Califfato. Gli Stati Uniti hanno, in principio, sostenuto soprattutto i peshmerga, i combattenti curdi irakeni tradizionali alleati degli U.S.A. nella guerra contro Saddam Hussein, per la liberazione, dall'occupazione del Califfo al-Baghdadi, dei territori appartenenti all'Iraq. Nonostante il drastico ridimensionamento, negli ultimi mesi, delle regioni su cui ancora insiste lo Stato Islamico e le notevoli perdite di uomini a questo inferte, sino al recente e decisivo attacco a Raqqa, sua capitale e fondamentale avamposto dei jihadisti, avviato il 22 settembre 2017, allorquando i "dissapori" tra U.S.A. e Russia sono stati strumentalmente e solo formalmente accantonati, le esclusive finalità delle potenze occidentali sono di mantenere il controllo delle aree di interesse geopolitico. Alla definitiva liberazione della città simbolo dell'oppressione dei popoli in Medio Oriente, attraverso un'opera di bonifica di Raqqa dalla residua presenza di milizie dell'ISIS, sta prendendo parte una coalizione (da intendere in senso lato, poiché operante su diversi fronti della Siria) composta da combattenti curdi, dall'esercito arabosiriano, dall'aviazione russa e dagli Hezbollah libanesi. Quanto al contributo degli U.S.A. alla radicale estirpazione del Califfato, l'attività nordamericana è esclusivamente funzionale a pianificare nuove strategie di attacco al governo di Bashar al-Assad e a garantirsi, di conseguenza, il controllo nella medesima area siro-irakena che si sta sottraendo al Daesh.

La crisi mediorientale, delineatasi a partire dal 2014, ha posto in evidenza l'esistenza di due coalizioni contrapposte: da un lato, Turchia, Arabia Saudita, Qatar (i principali finanziatori dell'ISIS) e l'alleanza Stati Uniti e Israele; e, dall'altro, una coalizione sciita, che vede, quali attori principali, l'Iran, la Siria di Bashar al-Assad e le organizzazioni libanese di Hezbollah e palestinesi presenti in Libano.

Gli "attacchi all'Occidente", perpetrati in città europee o frequentate da turisti occidentali, nell'attuale fase, inaugurata dagli attentati di Parigi del 7 gennaio 2015 e da ultimo scandita da quelli compiuti a Barcellona,

\footnotetext{
${ }^{41}$ Si tratta dell'Unità di Protezione delle Donne, una brigata interamente femminile che svolge un ruolo prioritario nella guerra di liberazione del Rojava dall'occupazione dell'ISIS.
} 
il 17 agosto 2017, e a Londra, il 15 settembre 2017, intravediamo una vera e propria strategia della tensione, analoga a quella pianificata in Italia e posta in essere nel periodo compreso tra le stragi di Piazza Fontana, il 12 dicembre 1969, e della stazione di Bologna, il 2 agosto 1980.

E, se in passato l'obiettivo locale era quello di creare una situazione di ingovernabilità, finalizzata, quantomeno, a un irrigidimento statale in senso autoritario, se non a un vero e proprio intervento militare golpista, le attuali finalità sovranazionali sono quelle di alimentare, strumentalizzando il senso di insicurezza collettiva, il sentimento di islamofobia e di intolleranza nei confronti dei migranti.

Il risultato della strategia della tensione, attuata a partire dalla seconda decade del nuovo millennio, è quello di rinnovare i presupposti, da un lato, per il dilagare di legislazioni emergenziali tese all'esclusione dei migranti e al ridimensionamento della concessione della protezione internazionale per i richiedenti asilo, nonché alla repressione interna ai singoli Stati di chi esprime dissenso ${ }^{42}$, e, dall'altro, per il perpetuarsi delle logiche di intervento e di ingerenza a livello globale.

Si ridefiniscono, nell'ambito degli U.S.A. e dell'U.E., più raffinate forme di controllo dei "cittadini" e di contenimento dei flussi migratori e, sul piano internazionale, nuove fonti di legittimazione alle strategie di aggressione militare statunitense ed europea nelle aree di interesse geopolitico.

\section{RIFERIMENTI}

A. STARACE, I diritti dei prigionieri islamici, in Innovazione e diritto, n. 4, 2007, 80 ss.

A.J. MAGLIACANE, Un Trauerspiel. L'intimazione di Riccardo III, in Costituzione, economia, globalizzazione. Liber Amicorum in onore di Carlo Amirante, Napoli, 2013, 399 ss.

B. PIATTOLI, Principio di proporzionalità UE e trattamento dei dati personali nella lotta al terrorismo, in Dir. pen. e proc., n. 7, 2015, 885 ss.

B. PIATTOLI, Principio di proporzionalità UE e trattamento dei dati personali nella lotta al terrorismo, cit., 885.

C. AMIRANTE, Il futuro dell'Unione Europea dopo i referendum: tra economia, espertocrazia e democrazia, in S. GAMBINO, Trattato che adotta una Costituzione per l'Europa, Costituzioni nazionali, diritti fondamentali, Milano, 2006, 169 ss.

C. AMIRANTE, Union Européenne et libertés économiques entre marché, état et démocratie, in L'architecture du droit. Mélanges en l'honneur du Professeur Michel Troper, Economica, 2006, 35 ss.

Consiglio europeo straordinario, Conclusioni della Presidenza e Piano di Azione, Bruxelles, 21 settembre 2001, in Rivista Italiana di Diritto Pubblico Comunitario, 2001, n. 5, 895 ss., con nota di M. BONINI, Oltre lo «Stato-nazione» per una politica europea di «lotta» al terrorismo.

Consiglio europeo straordinario, Conclusioni della Presidenza e Piano di Azione, Bruxelles, 21 settembre 2001, cit., 895-896.

Consiglio europeo straordinario, Conclusioni della Presidenza e Piano di Azione, Bruxelles, 21 settembre 2001, cit., 897-898.

\footnotetext{
${ }^{42}$ Un esempio, in tal senso, è offerto, in Italia, dai decreti legge 17 febbraio 2017, n. 13, e 20 febbraio 2017, n. 14, convertiti, rispettivamente, nella L. 13 aprile 2017, n. 46, e nella L. 18 aprile 2017, n. 48 (cc.dd. «decreti Minniti») su sicurezza e immigrazione, tesi al controllo, da un lato, di cittadini italiani e comunitari e, dall'altro, di migranti; cfr., sul punto, U. Nazzaro, I decreti "immigrazione" e "sicurezza" del 2017: considerazioni critiche, in Riv. pen., n. 7/8, 2017, pp. 599 ss.
} 
D. ALBANESE, Le motivazioni della Corte d'Assise di Milano sul "caso Fatima": spunti di riflessione su terrorismo internazionale e organizzazione di trasferimenti ex art. 270-quater.1 c.p., in Dir. pen. cont., 28 marzo 2017, www.penalecontemporaneo.it.

D. ALBANESE, Partecipazione all'associazione con finalità di terrorismo 'Stato Islamico': una pronuncia di condanna della Corte d'Assise di Milano, in Dir. pen. cont., 21 ottobre 2016, www.penalecontemporaneo.it.

D. RUSSO, Lotta al terrorismo internazionale e diritti umani: le "extraordinary renditions" nella giurisprudenza di Strasburgo, in Legisl. pen., n. 2, 2013, 319 ss.

F. MARONE, Il nuovo volto del terrorismo jihadista, in La rivista il Mulino, 2, 2015, 293 ss.

F. POLINO, Il contrasto alle nuove forme di terrorismo internazionale, in www.magistraturaindipendente.it, 6 marzo 2017.

J.R. CAPELLA, La globalizaciòn: ante una encrucijada politico-jurìdica, in Derecho y justicia en una sociedad global. Law and justice in a global society (Anales de la Càtedra Francisco Suàrez), Granada, España, Mayo 2005, 13 ss.

M. BONINI, La «lotta» al terrorismo: il quadro giuridico internazionale e comunitario.

M. BONINI, Oltre lo «Stato-nazione» per una politica europea di «lotta» al terrorismo, cit., 901.

M. DELMAS-MARTY, Studi giuridici comparati e internazionalizzazione del diritto, Torino, 2004, 23; C. FILOSA, L'ONU: mediazione irrigidita. Una pretesa universalità del particolare dominio di classe, in REDLINK (a cura di), L'ONU e «i signori della pace», Milano, 2004, 90 ss.; G. POOLE, Cosa fare dell'ONU?, in REDLINK (a cura di), L'ONU e «i signori della pace», cit., 98 ss.; S. SERINO, Una replica a Gordon Poole, in REDLINK (a cura di), L'ONU e «i signori della pace», cit., 107 ss.

M. FLORES, Le domande che non ci poniamo, in La rivista il Mulino, cit., 318 ss.

M. MANDEL, Guerre illegali, danni collaterali e crimini contro l'umanità: il ruolo della legge contro i crimini internazionali dal Kosovo all'Iraq ed oltre, in Crit. dir., n. 1-2-3, 2004, 22.

M. MANDEL, R2P \& ICC v. UNC: The Responsibility to Protect and the International Criminal Court versus the Charter of the United Nations, in Costituzione, economia, globalizzazione. Liber Amicorum in onore di Carlo Amirante, cit., 1375 ss.

M. MANNO, L'ONU e la questione palestinese, in REDLINK (a cura di), L'ONU e «i signori della pace», cit., 117 ss.

M. MIRAGLIA, Lotta al terrorismo e diritti dei prigionieri: la Corte Suprema U.S.A. richiama al rispetto dei principi costituzionali, in Dir. pen. e proc., n. 11, 2004, 1426.

M. MIRAGLIA, Paura e libertà (Legislazione antiterrorismo e diritti di difesa negli Stati Uniti), in Ques. giust., n. 2-3, 2004, 304 ss.

M. MIRAGLIA, Una nuova normalità: metamorfosi della giustizia penale statunitense dopo l'11 settembre, in Cass. pen., n. 9, 2005, 2832-2834.

M. RATNER, E. RAY, Prigionieri di Guantanamo. Quello che il mondo deve sapere, Bologna, 2005. 
M. TORREALTA (a cura di), Guerra e informazione. Un'analisi fuori da ogni schieramento, Sperling \& Kupfer, 2005.

N. BARTONE, Mandato di arresto europeo e tipicità nazionale del reato, Milano, 2003, con prefazione di G. VASSALLI, 296.

P. COLLIER, D. DOLLAR, Globalizzazione, crescita economica e povertà. Rapporto della Banca Mondiale, Bologna, 2003.

P. PICONE, La guerra contro l'Iraq e le degenerazioni dell'unilateralismo, in Rivista di diritto internazionale, 2003, I, 347.

P. POLICASTRO, 'On The Reconstruction of the Legal Strength of the Constitution in a World in transition. Multi-Level Constitutionalism towards Multi-level Democracy', in J. NERGELIUS, P. POLICASTRO, K. URATA (a cura di), Challenges of Multilevel Constitutionalism, Proceedings of IVR $21^{\text {st }}$ World Congress, Lund 2003, Krakòw; P. POLICASTRO, A new Garment for an Old Question: 'A Clash between Man's Rights and Citizens' Rights in the Enlarged Europe', in J. NERGELIUS (a cura di), Nordic and other European Constitutional Traditions, Leiden/Boston, 2006, 66.

P.L. BERGEN, Holy war, Inc. Osama bin Laden e la multinazionale del terrore, Milano, 2001.

R. BERTOLESI, Indottrinare al martirio non è reato di associazione con finalità di terrorismo, in Dir. pen. cont., 23 gennaio 2017, www.penalecontemporaneo.it.

REDLINK, L'ONU e «i signori della pace», cit., 70.

S. LICCIARDELLO, Nuove norme antiterrorismo in Italia, in www.sicurezzanazionale.gov.it., 9 settembre 2016.

U. Nazzaro, I decreti "immigrazione" e "sicurezza" del 2017: considerazioni critiche, in Riv. pen., n. 7/8, 2017, pp. 599 ss.

U. NAZZARO, Il diritto penale del nemico tra delitto di associazione politica e misure di contrasto al terrorismo internazionale, Giannini (Quaderni dell'Accademia Pontaniana, n. 62), Napoli, 2016.

U. NAZZARO, Le misure di contrasto al terrorismo internazionale alla luce della Legge 17 aprile 2015, n. 43, in Riv. pen., n. 10, 2015, 822 ss.

V. MONETTI, La legislazione antiterrorismo dopo l'11 settembre: il contesto internazionale e l'Italia, in Ques. giust., n. 1, 2002, 51. 